\title{
Ingestão calórica e alta hospitalar em cães e
}

\section{gatos}

\section{Energy consumption and hospital discharge in dogs and cats}

\section{Ingestión calórica y el alta en hospitalización de perros y gatos}

\section{Aulus Cavalieri Carciofi'; Valéria Oliveira Fraga²; Márcio Antônio Brunetto ${ }^{3}$}

Departamento de Clínica e Cirurgia Veterinária da Faculdade de Ciências Agrárias e Veterinárias da Universidade Estadual Paulista, Campus de Jaboticabal, SP, Brasil

\section{Resumo}

Objetivo: Investigar a correlação entre ingestão calórica e tempo de internação com a alta ou o óbito de cães e gatos. Material e Método: Animais internados no Hospital Veterinário da Faculdade de Ciências Agrárias e Veterinárias da Universidade Estadual Paulista (HV-FCAV/UNESP), Campus de Jaboticabal, SP, por qualquer motivo de doença no pré ou pós-cirúrgico, tiveram estimada sua necessidade energética de manutenção (NEM): $132 \times$ (peso corporal) ${ }^{0,75} \mathrm{kcal}$ por dia para cães; $70 \mathrm{x}$ (peso corporal) kcal por dia para gatos. Receberam alimento comercial super-premium, suporte enteral e parenteral. Foram incluídos 279 animais (223 cães e 56 gatos). Resultados: Tiveram alta hospitalar 76,7\% dos pacientes, cuja ingestão percentual média da NEM foi de $71,12 \%$. Já os que vieram a óbito ingeriam apenas $33,97 \%$ da NEM ( $<<0,001)$. Os animais do grupo Alta permaneceram 7,18 dias no hospital, enquanto os do grupo Óbito 5,45 dias $(\mathrm{p}=0,16)$. Dentre os animais que receberam de $0 \%$ a $33 \%$ da NEM, 38\% tiveram alta e $62 \%$ vieram a óbito; dos que receberam entre $34 \%$ e $66 \%$ da NEM, $84 \%$ tiveram alta e $16 \%$ vieram a óbito; para os que receberam mais de $67 \%$ da NEM, $89 \%$ tiveram alta e $11 \%$ vieram a óbito, demonstrando menor mortalidade nas faixas de maior balanço calórico ( $p<0,001)$. Nestas faixas, os animais permaneceram mais tempo hospitalizados $(\mathrm{p}<0,002)$, o que também pode ser verificado pela associação positiva entre ingestão de calorias e dias de internação $(R=0,21 ; p<0,05)$. Conclusões: Verificou-se que dentre os animais para os quais administrou-se maior quantidade de calorias o número de alta e tempo de permanência hospitalares foram maiores.

Palavras-chave: Hospitalização. Tempo de internação. Alta do paciente. Terapia nutricional. Avaliação nutricional. Necessidade energética. Ingestão calórica. Cães. Gatos.

'Professor Assistente Doutor. Departamento de Clínica e Cirurgia Veterinária da FCAV/UNESP, Campus de Jaboticabal. CRMV-SP 6707

${ }^{2}$ Residente em Nutrição Clínica de Cães e Gatos da FCAV/UNESP, Campus de Jaboticabal. CRMV-SP 13596

${ }^{3}$ Residente em Nutrição Clínica de Cães e Gatos da FCAV/UNESP, Campus de Jaboticabal 


\section{Introdução e Revisão de Literatura}

Nas últimas duas décadas houve um aumento das informações científicas a respeito da nutrição do animal enfermo. Este conhecimento, no entanto, ainda não faz parte da rotina médica de clínicas e hospitais veterinários, que usualmente não consideram a nutrição uma parte do tratamento médico veterinário (REMILLARD, et al. ${ }^{1}, 2000$ ). A má-nutrição de animais internados é mais comum do que usualmente se reconhece. Em estudo envolvendo 276 cães e 821 dias de hospitalização, Remillard et al. ${ }^{2}$ (2001) relataram que em apenas $27 \%$ dos dias os animais ingeriram alimento suficiente para atingirem balanço calórico positivo. Entre as causas que levaram ao balanço energético negativo listaram a recusa do animal em se alimentar ou anorexia (43\%), prescrição de jejum (34\%) e prescrição dietética incorreta por parte dos médicos veterinários (22\%).

Geralmente os pacientes internados diminuem seu apetite em decorrência da doença, estresse da hospitalização, ambiente e pessoas estranhos e dor no pós-operatório (BOULCOTT $\left.{ }^{3}, 1967\right)$. Entretanto, é justamente neste momento que precisam de uma atenção nutricional especial, pois em resposta às referidas mudanças, observam-se alterações metabólicas que resultam em aumento das necessidades nutricionais, na taxa metabólica (hipermetabolismo) e persistente perda de nitrogênio associada a um balanço nitrogenado negativo. Tais fatos, se sobrepõem ou pioram a deficiência nutricional pré-existente (BUTTERWICK; TORRANCE $\left.{ }^{4}, 1995\right)$. Os efeitos da má-nutrição calórico-protéica tendem a ser específicos para cada tecido e podem tornar-se generalizados quanto maior for a demora em sua correção. Longos períodos de privação alimentar culminam em grande mobilização de aminoácidos, que são utilizados na síntese de DNA e RNA, além da produção de proteínas de fase aguda e de energia (gliconeogênese), agravando o estado de desnutrição (SEIM III; BARTGES 5 , 2003).

A assistência nutricional ao paciente hospitalizado tem como objetivos manter ou evitar o decréscimo da imunocompetência, da síntese e reparação tecidual e do metabolismo intermediário de drogas, as três prin- cipais consequiências da desnutrição. Entende-se como desnutrição ou má-nutrição o consumo insuficiente de calorias, proteínas e oligoelementos necessários para o metabolismo tecidual normal, o que prejudica diretamente o manejo médico ou cirúrgico do paciente (REMILLARD, et al. ${ }^{2}, 2001$; BATTAGLIA $^{6}$, 2001).

O correto manejo nutricional do animal hospitalizado depende de uma adequada coleta de informações nutricionais durante a anamnese e o exame físico (incluindo a condição ou escore corporal), e da realização de exames laboratoriais específicos, quando necessário. É preciso estruturar protocolos e procedimentos internos que permitam a definição das necessidades calóricas do animal, do tipo de alimento e via de administração e da quantidade a ser fornecida. Mecanismos de acompanhamento e registros diários do consumo efetivo de alimentos e da produção de fezes são também fundamentais.

Os protocolos devem permitir ainda a identificação precoce de quando e como intervir nutricionalmente em casos mais graves. Animais que durante 24 ou 48 horas não apresentem consumo voluntário de suas necessidades energéticas entrarão em balanço calórico-protéico negativo, devendo receber intervenção nutricional enteral ou parenteral (REMILLARD, et al. ${ }^{1}$, 2000). A cada dia negligenciado, aumentam significativamente os riscos de complicações e mortalidade, complicando-se as desordens fisiológicas e dificultando o tratamento da doença primária (DEVEY, et al. ${ }^{7}, 1995$, CASE, et al. $\left.{ }^{8}, 1995\right)$.

O presente estudo investigou a correlação e a associação entre a ingestão calórica de cães e gatos hospitalizados com o tempo de internação e a alta ou óbito dos animais, bem como levantou as principais doenças relacionadas à anorexia.

\section{Material e Método}

Participaram do estudo cães e gatos internados no Hospital Veterinário “Governador Laudo Natel”, da Faculdade de Ciências Agrárias e Veterinárias da Universidade Estadual Paulista (HV - FCAV/UNESP), Campus de Jaboticabal, SP. Os animais foram hospitalizados por decisão dos Serviços de Clínica Médica, 
Clínica Cirúrgica e Obstetrícia e tiveram seu manejo alimentar sob responsabilidade do Serviço de Nutrição Clínica. Foram incluídos animais com qualquer doença primária e no pré ou pós-cirúrgico. A coleta dos dados foi realizada num período de nove meses.

\section{Protocolo Alimentar}

Todo animal, ao ser internado, foi pesado e teve calculada a sua Necessidade Energética de Manutenção (NEM) em kcal de energia metabolizável por dia, por meio das fórmulas:
NEM $=132 \times(\text { peso vivo })^{0,75} \mathrm{kcal}$ por dia para cães $\left(\mathrm{NRC}^{9}, 1985\right)$

$\mathrm{NEM}=70 \mathrm{x}$ peso vivo $\mathrm{kcal}$ por dia para gatos $\left(\mathrm{NRC}^{10}\right.$, 1986)

Os pacientes ficaram alojados em canis ou gatis individuais. Foram inicialmente alimentados com ração comercial seca tipo super-premium (Receita da Natureza ${ }^{\circledR} *$, Tabela 1), exceto nos casos em que houve prescrição médica de jejum devido a vômito, diarréia ou processo cirúrgico. Mesmo sob jejum forçado, os animais foram incluídos no estudo, sendo computado seu

Tabela 1 - Composição de rótulo dos alimentos empregados no experimento

\begin{tabular}{|c|c|c|c|c|c|}
\hline \multirow{2}{*}{ Nutriente $^{1}$} & \multicolumn{5}{|c|}{ Alimento industrializado utilizado } \\
\hline & $\begin{array}{c}\text { Para cães } \\
\text { normometabólicos }{ }^{2}\end{array}$ & $\begin{array}{c}\text { Para cães } \\
\text { hipermetabólicos }^{3}\end{array}$ & $\begin{array}{c}\text { Para cães } \\
\text { hiperazotêmicos }\end{array}$ & $\begin{array}{c}\text { Para } \\
\text { gatos }^{5}\end{array}$ & $\begin{array}{l}\text { Alimento } \\
\text { úmido }^{6}\end{array}$ \\
\hline MS, $\%$ & 88 & 88 & 88 & 90 & 20 \\
\hline $\mathrm{MM}, \%$ & 8 & 8 & 8 & 8,2 & 3 \\
\hline $\mathrm{PB}, \%$ & 25 & 25 & 18 & 31 & 8 \\
\hline $\mathrm{EE}, \%$ & 15 & 15 & 8 & 8 & 5 \\
\hline $\mathrm{FB}, \%$ & 3 & 3 & 5 & 4,5 & 3 \\
\hline Cálcio, \% & 1,3 & 1,3 & 1,3 & 2,5 & 0,5 \\
\hline Fósforo, \% & 0,7 & 0,7 & 0,8 & 1 & 0,3 \\
\hline $\mathrm{ENN}, \%$ & 37 & 37 & 49 & 38,3 & 1 \\
\hline $\mathrm{EM}, \mathrm{kcal} / 100 \mathrm{~g}$ & 344 & 344 & 320 & 280 & 67,7 \\
\hline
\end{tabular}

MS- Matéria Seca; MM- Matéria Mineral; PB- Proteína Bruta; EE- Extrato Etéreo; FB- Fibra Bruta; ENN- Extrativos Não-Nitrogenados; EM- Energia Metabolizável em kcal calculada para cães como (PB x 3,5) + (EE x 8,5) + (ENN x 3,5), segundo NRC (1985), e para gatos como $[(\mathrm{PB} \times 5,65)+(\mathrm{EE} \times 9,4)+(\mathrm{ENN} \times 4,15) \times 0,99]-126$, segundo (KELLY, 1996).

${ }^{2}$ Lista de ingredientes: carne de frango, arroz integral, gordura de frango, óleo de canola, polpa de beterraba, cloreto de sódio, tocoferol, ácido cítrico, essência de alecrim, vitaminas A, B1, B2, B6, B12, D3, E, cloreto de colina, niacina, ácido pantotênico, fosfato bicálcico, sulfato de ferro, proteinato de zinco, iodato de potássio, selenito de sódio, cloreto de potássio, sulfato de manganês.

${ }^{3}$ Lista de ingredientes: carne de frango, arroz integral, gordura de frango, óleo de canola, polpa de beterraba, cloreto de sódio, tocoferol, ácido cítrico, essência de alecrim, vitaminas A, B1, B2, B6, B12, D3, E, cloreto de colina, niacina, ácido pantotênico, fosfato bicálcico, sulfato de ferro, proteinato de zinco, iodato de potássio, selenito de sódio, cloreto de potássio, sulfato de manganês.

${ }^{4}$ Lista de ingredientes: carne de frango, arroz integral, gordura de frango, óleo de canola, polpa de beterraba, cloreto de sódio, tocoferol, ácido cítrico, essência de alecrim, vitaminas A, B1, B2, B6, B12, D3, E, cloreto de colina, niacina, ácido pantotênico, fosfato bicálcico, sulfato de ferro, proteinato de zinco, iodato de potássio, selenito de sódio, cloreto de potássio, sulfato de manganês.

${ }^{5}$ Lista de ingredientes: farinha de peixe, milho integral moído, arroz quebrado, farinha de carne de frango, hidrolisado de frango, farinha de glúten de milho, farinha de soja, gordura animal estabilizada, ácido fosfórico, cloreto de sódio, levedura seca de cervejaria, corante, cloreto de potássio, carbonato de cálcio, sulfato de zinco, premix vitamínico, sulfato de ferro, taurina, sulfato de cobre, sulfato de manganês, menadiona bissulfito de sódio, iodeto de potássio, selenito de sódio.

${ }^{6}$ Lista de ingredientes: carne de frango, miúdos bovinos, cloreto de sódio, carbonato de cálcio, fosfato bicálcico, premix vitamínico mineral, carragena, água.

*Mogiana Alimentos S.A. (Guabi) 
consumo como zero e, portanto, em balanço energético negativo.

A quantidade de alimento a ser administrada foi calculada considerando-se a NEM do paciente e a energia metabolizável (EM) do alimento. Esta última foi estimada a partir da composição de rótulo dos alimentos pelas fórmulas:

$\mathrm{EM}=[($ proteína bruta x 3,5) $+($ extrato etéreo $\mathrm{x}$ $8,5)+($ extrativos não-nitrogenados x 3,5)] kcal por 100 gramas para alimentos destinados a cães (NRC, 1985)

$\mathrm{EM}=[($ proteína bruta x 5,65) $+($ extrato etéreo x 9,4) + (extrativos não-nitrogenados x 4,15) ] x 0,99$126 \mathrm{kcal}$ por 100 gramas para alimentos secos destinados a gatos (KELLY $\left.{ }^{11}, 1996\right)$

$\mathrm{O}$ alimento foi oferecido duas vezes ao dia, às 09hs. e às 16hs., permanecendo disponível ao animal até a próxima alimentação. Em uma planilha apropriada foram computadas as quantidades oferecidas em cada refeição e uma estimativa do consumo de alimentos, em porcentagem.

Nos casos em que o alimento oferecido não foi consumido ou o foi em baixa quantidade, utilizaram-se palatabilizantes como ração úmida, água morna ou creme de leite e, eventualmente, comida caseira. Quando este artifício não contornava a anorexia, praticava-se a ingestão forçada, com a colocação de alimento diretamente na boca do animal. Havendo insucesso, partiase, então, para o suporte nutricional enteral por meio da colocação de sonda nasogástrica, esofágica ou gástrica, dependendo da situação clínica do paciente. Nos pacientes em que a via gastroentérica apresentou-se inviável devido a vômitos ou recuperação pós-cirúrgica, instituiu-se suporte nutricional parenteral parcial periférico, constituído por glicose a $50 \%$, solução de aminoácidos a $10 \%$, eletrólitos, complexo B, arginina e vitamina $\mathrm{K}$.

\section{Análise dos Resultados}

Para a tabulação e a análise dos resultados, os animais foram classificados e separados de acordo com o sucesso da terapia e da ingestão calórica. Quanto ao sucesso da terapia, foram separados em dois grupos, os que tiveram alta (Grupo Alta) e os que tiveram óbito (Grupo Óbito) por morte natural ou eutanásia. Em relação à ingestão de calorias, não se diferenciou entre consumo voluntário, forçado, enteral ou suporte nutricional parenteral. Os animais foram classificados em três faixas de ingestão calórica, conforme a NEM: Consumo 1 de $0 \%$ a $33 \%$; Consumo 2 de $34 \%$ a $66 \%$; Consumo 3 de $67 \%$ a $100 \%$.

Os pacientes foram classificados de acordo com a ingestão da Necessidade Energética de Repouso (NER) em duas faixas: Grupo Balanço Negativo, para ingestão inferior à NER; Grupo Balanço Positivo, para ingestão igual ou superior à NER. Esta foi calculada, tanto para cães como para gatos, pela fórmula:

$\mathrm{NER}=70 \mathrm{x}(\text { peso vivo })^{0,75}$, kcal por dia $\left(\mathrm{KLEIBER}^{12}, 1932\right)$

A inclusão do animal nas faixas de consumo, na primeira e na segunda análises, considerou a ingestão média do paciente durante o período em que esteve hospitalizado.

Registrou-se também o número de dias em que os pacientes ficaram internados. Os animais agrupados distribuíram-se nas seguintes faixas: Internação 1, quando permaneceram de 1 a 5 dias; Internação 2, quando permaneceram de 6 a 15 dias; Internação 3, para 16 dias ou mais.

\section{Análise Estatística}

A análise estatística foi feita com o auxílio do software estatístico SAS (SCHLOTZHAUER; LITTELL $^{13}$, 1997). Utilizou-se o teste t para comparar a média de consumo de alimentos e a média de dias de internação entre os grupos Alta e Óbito. Realizou-se o teste de Qui-quadrado e a correlação de Spearman para verificar a dependência entre os grupos Alta e Óbito e as faixas de consumo, em relação à NEM e à NER. Os mesmos testes foram realizados para correlacionar as faixas de consumo e as faixas de dias de internação. Os resultados foram considerados estatisticamente significativos quando $\mathrm{p} \leq 0,05$. 


\section{Resultados}

O estudo incluiu 279 animais, 223 cães e 56 gatos, num total de 1.760 dias de observação. Em relação à NER, dos animais avaliados, 64,1\% apresentaram balanço energético positivo e $35,9 \%$, balanço energético negativo. Quanto à via de alimentação, a maioria apresentou ingestão voluntária, como pode ser visto na Tabela 2.

Tabela 2 - Tipo de suporte nutricional empregado durante o experimento

\begin{tabular}{lcc}
\hline \multirow{2}{*}{ Via de alimentação } & \multicolumn{2}{c}{ Animais } \\
\cline { 2 - 3 } & Total & $\%$ \\
\hline Alimentação voluntária & & \\
\cline { 2 - 3 } & 246 & 88,2 \\
Sonda nasogástrica & 17 & 6,1 \\
Sonda esofágica & 4 & 1,4 \\
Sonda gástrica & 3 & 1,1 \\
Nutrição parenteral & 9 & 3,2 \\
\hline
\end{tabular}

'Inclui a ingestão forçada e o uso ou não-uso de palatabilizantes

Tiveram alta hospitalar (Grupo Alta) 214 animais, representando $76,7 \%$ dos pacientes, enquanto os outros $65(23,3 \%)$ integraram o Grupo Óbito. A ingestão calórica, em porcentagem da NEM, verificada para o Grupo Alta foi de $71,12 \%$, enquanto para o Grupo Óbito foi de $33,97 \%$, havendo uma diferença estatisticamente significativa pelo teste $\mathrm{t}(\mathrm{p}<0,001)$. Em relação aos dias de internação, os animais do grupo Alta permaneceram, em média, 7,18 dias no hospital, enquanto os do Grupo Óbito ficaram 5,45 dias, resultados estatisticamente iguais pelo teste $\mathrm{t}(\mathrm{p}=0,16)$.

O teste de Qui-quadrado verificou dependência estatística significativa entre as faixas de consumo referentes à NEM acompanhadas dos grupos Alta e Óbito e às faixas de dias de internação. Estes dados encontram-se na Tabela 3.
Tabela 3 - Resultados do teste de Qui-quadrado para o sucesso da terapia, faixas de internação e as faixas de consumo da NEM*

\begin{tabular}{lcc}
\hline Faixa de internação & \multicolumn{2}{c}{ Faixa de consumo da NEM } \\
\cline { 2 - 3 } & Quadrado & P \\
\hline Alta ou óbito & 63,49 & 0,001 \\
Dias de internação & 16,46 & 0,002 \\
\hline
\end{tabular}

*NEM = Necessidade Energética de Manutenção

Considerando a ingestão calórica em porcentagem da NEM, a faixa de Consumo $1(0 \%$ a $33 \%)$ apresentou $38 \%$ de animais que tiveram alta e $62 \%$ que vieram a óbito; a faixa de Consumo 2 (34\% a 66\%) incluiu $84 \%$ de animais que tiveram alta e $16 \%$ que vieram a óbito; na faixa de Consumo 3 (acima de 67\%), 89\% dos animais tiveram alta e $11 \%$, óbito. Portanto, nas faixas de maior consumo a mortalidade dos animais foi menor, resultado significativo pelo teste de Qui-quadrado $(p<0,001)$. A associação entre ingestão de alimentos e Alta ou Óbito, avaliada pela correlação de Spearman, obteve $R=-0,40$ e $p<0,05$. Esta associação negativa pode indicar que quanto maior a ingestão calórica, menor a mortalidade.

Quanto aos dias de internação, na faixa de Consumo $1,78 \%$ dos animais permaneceram internados até 5 dias (Internação 1), 20\% de 6 a 15 dias (Internação 2) e 2\%, 16 dias ou mais (Internação 3). Dentre os animais que mais se alimentaram (faixa de Consumo 3) $50 \%$ permaneceram até 5 dias hospitalizados, $39 \%$ de 6 a 15 dias e $11 \%$, 16 dias ou mais. Portanto, nas faixas de maior consumo os animais permaneceram mais tempo hospitalizados, resultado significativo pelo Qui-quadrado $(p<0,002)$. A associação entre a ingestão de alimentos e as faixas de dias de internação, avaliada pela correlação de Spearman, obteve $R=0,21$ e $p<0,05$. Esta associação positiva pode indicar que quanto maior a ingestão calórica, maior o tempo de internação.

Foram levantadas as doenças que mais causaram hiporexia. Para esta análise, agruparam-se os animais das faixas de Consumo 1 e 2, que foram comparados com os da faixa de Consumo 3. Os resultados obtidos são apresentados na Tabela 4. 
Tabela 4 - Ocorrência percentual de afecções clínicas dentre os animais hospitalizados pertencentes às faixas de Consumo 1 e 2 (até $66 \%$ da NEM*), em comparação à faixa de Consumo 3 (acima de $67 \%$ da NEM)

\begin{tabular}{|c|c|c|}
\hline \multirow[b]{2}{*}{ Afecção } & \multicolumn{2}{|c|}{ Ocorrência da afecção (\%) } \\
\hline & $\begin{array}{l}\text { Faixa de } \\
\text { consumo } 3\end{array}$ & $\begin{array}{c}\text { Faixas de } \\
\text { consumo } 1 \text { e } 2\end{array}$ \\
\hline Nefropatias & 32,1 & 4,8 \\
\hline Hemoparasitoses & 14,6 & 7,2 \\
\hline Traumas & 8,8 & 46,4 \\
\hline Gastroenterites & 11,7 & 7,2 \\
\hline Cinomose & 7,3 & 4,8 \\
\hline Piometra & 6,6 & 7,2 \\
\hline Cardiopatias & 4,4 & 0,8 \\
\hline Doenças do trato urinário & 4,4 & 4,0 \\
\hline Neoplasias & 4,4 & 4,0 \\
\hline Diabetes mellitus & 2,9 & 0,8 \\
\hline Hepatopatias & 1,4 & 0,8 \\
\hline Afecções respiratórias & 1,4 & 1,6 \\
\hline Afecções neurológicas & - & 0,8 \\
\hline Afecções dermatológicas & - & 3,2 \\
\hline Intoxicações & - & 4,8 \\
\hline Megaesôfago & - & 1,6 \\
\hline
\end{tabular}

*NEM = Necessidade Energética de Manutenção

\section{Discussão}

Atualmente, no Brasil, raramente encontra-se algum hospital ou clínica veterinária que possua um rigoroso critério para o controle do consumo de alimentos ou para o fornecimento de calorias via nutrição intensiva para os animais internados. Alguns clínicos ainda acreditam que a intervenção nutricional não é tão necessária e que, instituindo-se uma terapia adequada, $\mathrm{o}$ apetite pode se regularizar em até 5 dias (BURKHOLDER ${ }^{14}$, 1995). Sabe-se hoje, no entanto, que a ingestão de alimentos apresenta muitos benefícios (CHANDRA $^{15}$, 1981; CRANE ${ }^{16}$, 1989; CHANDRA $^{17,18}$, 1992; FETTMAN; PHILLIPS ${ }^{19}$, 2000; REMILLARD, et al. ${ }^{2}$, 2001), e é fundamental no sucesso da terapia e recuperação do paciente (SIMPSON $^{20}$, et al., 1993; LEWIS, et al. ${ }^{21}$, 1990; HILL $^{22}$, 1994; CASE et al. ${ }^{8}$, 1995; TENNANT ${ }^{23}$, 1996; REMILLARD, et al. ${ }^{1}$, 2000). O suporte nutricional pode ser tão vital como qualquer outra terapia, como por exemplo, a fluidoterapia ou a antibioticoterapia (DEVEY, et al. ${ }^{7}, 1995$ ).

O único trabalho em cães encontrado na literatura científica investigando a relação entre ingestão calórica e alta hospitalar é o de Remillard et al. ${ }^{2}$ (2001). Os autores relatam que em apenas $26 \%$ dos dias os cães apresentaram balanço energético positivo (ingestão > 95\% da NER). No presente estudo observou-se que em $64,1 \%$ dos dias os animais tiveram balanço calórico positivo, número 2,46 vezes maior. Esta diferença talvez se deva ao fato de o estudo de Remillard et al. ${ }^{2}$ (2001) ter se realizado em 4 hospitais veterinários e as prescrições nutricionais nem sempre terem sido feitas por nutricionistas, enquanto que na presente pesquisa a alimentação dos animais ficou sob a responsabilidade do Serviço de Nutrição Clínica.

Por outro lado, os autores supracitados não encontraram relação estatística de dependência entre o escore de condição corporal e a ingestão calórica ou alta hospitalar. Por outro lado, o escore de condição física, que mede o grau de doença e comprometimento orgânico, apresentou relação tanto com a ingestão calórica $(\mathrm{p}<0,03)$ como com a alta hospitalar ( $\mathrm{p}<0,001)$. Da mesma forma, no presente experimento, os autores também encontraram associação estatística entre a ingestão calórica diária e a alta hospitalar $(\mathrm{p}<0,01)$. Discutem em seu trabalho que a anorexia éo principal fator causal do balanço energético negativo.

Pelos resultados do presente estudo, observa-se que o grupo de animais que apresentou maior ingestão calórica teve maior porcentagem de alta hospitalar e permaneceu mais tempo hospitalizado. Dessa forma, supõe-se que a manutenção de um balanço energético positivo auxilie no curso de terapias médicas mais longas e especiais, resultando em melhores respostas dos 
pacientes. Os animais em balanço energético negativo demonstraram maiores taxas de óbito, conseqüentemente permaneceram menos tempo hospitalizados. Mesmo não sendo possível separar os efeitos relativos à severidade da doença, pois animais debilitados e com comprometimento funcional tendem a ingerir menos alimento, resultando em maior mortalidade, esses resultados indicam que o fornecimento adequado de calorias conduz a um prognóstico mais favorável.

A literatura apresenta inúmeros fatores pelos quais a baixa ingestão calórico-protéica correlaciona-se a uma mortalidade maior. Ela é a causa mais comum de imunodeficiência em pacientes humanos. Segundo Johansen, et al. ${ }^{24}$ (2003), uma grande proporção de pacientes humanos hospitalizados está em estado de subnutrição. Relatam em seu trabalho que aproximadamente $40 \%$ das pessoas já estão subnutridas no momento em que são hospitalizadas e que mais de $75 \%$ dos pacientes permanecem por mais de uma semana internados para recuperar o peso corporal perdido. Carnevale et al. ${ }^{25}$ (1991) verificaram uma incidência de desnutrição ou subnutrição de $25 \%$ a $65 \%$, baseando-se no históri$\mathrm{co}$, nos efeitos fisiológicos da doença primária e em parâmetros bioquímicos dos pacientes caninos e felinos estudados. Na rotina clínica, é comum receber pacientes com histórico de hiporexia, ou mesmo anorexia, ha vários dias. Esta condição não pode ser desconsiderada pelo clínico, pois neste momento o paciente já adentra o consultório desnutrido, necessitando de intervenção nutricional imediata.

Na desnutrição, verifica-se um decréscimo progressivo da resposta imune do paciente, incluindo a resposta mediada por células, produção de IgA secretória, fagocitose, funcionamento do sistema-complemento, afinidade de anticorpos e produção de citoquinas (CHANDRA $^{15}, 1981$; CHANDRA $\left.{ }^{17,18}, 1992\right)$. Muitos processos mórbidos associados à anorexia prolongada podem causar prejuízos à mucosa gastroentérica, por alterações de perfusão e ou oxigenação, resultando na produção de substâncias que desencadeiam reações em cascata e determinando o comprometimento da mucosa gastrointestinal, das células imunes locais, da secreção de imunomediadores e a perda da barreira entérica protetora. Tal processo culmina com a entrada de antígenos na circulação sistêmica e compromete múltiplos órgãos e sistemas (SAKER ${ }^{26}$, 2004). Crowe et al. ${ }^{27}$, em 1997, enfatizaram a importância do uso precoce do suporte nutricional enteral de modo a prevenir depressão do sistema imune, decréscimo nos níveis de albumina sérica, catabolismo muscular, translocação bacterinana, sepse, falência múltipla de órgãos e óbito.

Para que haja uma adequada síntese e reparação de feridas, uma nutrição adequada é essencial. No local, são necessários aminoácidos e glicose para a síntese de colágeno e metabolismo celular; no sistema são necessários nutrientes para a síntese hepática de fibronectina, complemento e glicose, atividade muscular cardíaca e respiratória envolvidas no transporte de oxigênio e nutrientes para a área afetada $\left(\mathrm{CRANE}^{16}, 1989\right)$.

O efeito terapêutico de drogas é afetado pelo estado nutricional do animal. Sua absorção, transporte, metabolismo e excreção também podem ser alterados. Diminuição da biotransformação hepática, decréscimo das proteínas plasmáticas envolvidas no transporte das drogas e diminuição do fluxo sangüíneo renal são conseqüências da desnutrição calórico-protéica que podem interferir na farmacocinética das drogas (FETTMAN; PHILLIPS $^{19}$, 2000).

Algumas afecções clínicas estão mais fortemente associadas à hiporexia. Nefropatias, hemoparasitoses, gastroenterites e piometra foram as mais freqüentemente verificadas dentre os animais hiporéticos, ao passo que os traumas somaram $46,4 \%$ dos animais normoréticos (Tabela 4).

Um sistema adequado de acompanhamento e registro da ingestão calórica é fundamental no manejo nutricional de animais doentes. Apesar de se considerar que os animais hospitalizados possam não ter benefícios adicionais com a ingestão de uma quantidade maior de alimento do que a NER, neste estudo optou-se oferecer uma quantidade que atendesse sua NEM, pois fatores relativos à doença podem aumentar o gasto energético em 1,05 a 1,2 vezes nos casos de traumas simples; em 1,2 a 1,3 vezes para múltiplas fraturas e queimaduras severas, podendo atingir o dobro das necessidades em traumas cranianos severos e estágios terminais de alguns tipos de câncer (DONOGHUE; KRONFELD $^{28}$, 1994). 


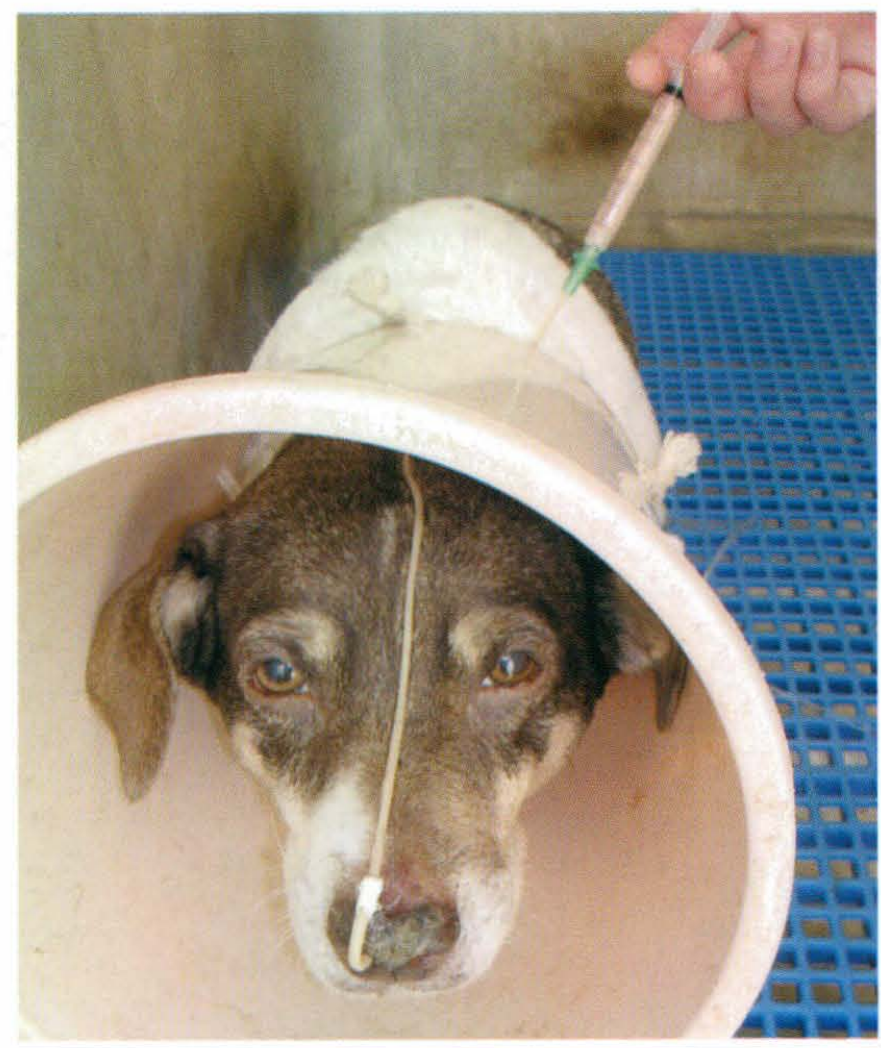

Figura 1 - Cão SRD recebendo alimentação enteral via sonda nasogástrica

O fornecimento de calorias e proteínas via nutrição intensiva é fundamental e deve constar do plano terapêutico de uma clínica ou hospital veterinário. No presente estudo, $12 \%$ dos animais receberam este tipo de suporte, o que foi responsável, em parte, pelo maior número de pacientes em balanço energético positivo e pelo sucesso de alguns procedimentos terapêuticos. Animais que não podem se alimentar devido a vômitos, diarréias intensas, atonia esofágica ou cirurgias do trato digestório devem receber nutrição parenteral. Como pode constatado na Tabela 2, apenas 3,2\% dos animais receberam esse tipo de suporte nutricional no presente estudo, que se deve exclusivamente a seu alto custo, o que impediu os proprietários de autorizarem seu uso.

Para Simpson e Elwood ${ }^{29}$ (1994), animais anoréticos com o trato gastrointestinal funcional devem ser prioritariamente alimentados via sonda nasogástrica, esofágica ou gástrica. A sonda nasogástrica foi o recurso mais empregado durante este estudo (Figura 1). Essa deve ser a primeira opção quando o suporte nutricional for necessário por um período de até 7 dias. Apresenta

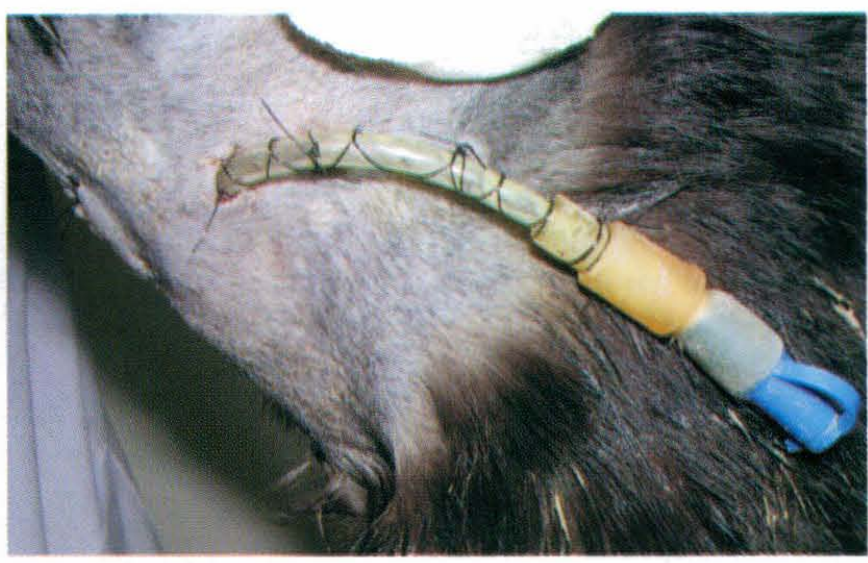

Figura 2 - Sonda esofágica aplicada em gato com fratura de mandíbula

fácil colocação, baixo custo, poucas complicações e dispensa o uso de anestésicos, muitas vezes contra-indicados em algumas apresentações clínicas (ABOOD; BUFFINGTON $\left.^{30,31}, 1991,1992\right)$. Quando existe a necessidade de um suporte mais longo, em decorrência da afecção primária, o emprego de sondas gástrica ou esofágica é necessário. O suporte nutricional enteral com o emprego de sonda esofágica é um método bem-tolerado pelos pacientes, fácil, prático, seguro, econômico e fisiológico, apresentando mínima morbidade (BATAGLIA $^{6}, 2001$; LEVINE, et al. ${ }^{32}$, 1997). Tal recurso tem sido freqüentemente empregado pelo Serviço de Nutrição Clínica, com resultados satisfatórios (Figura 2). Em situações, nas quais não se espera o retorno da capacidade de deglutição, a sonda gástrica passa a ser a melhor opção (Figura 3).

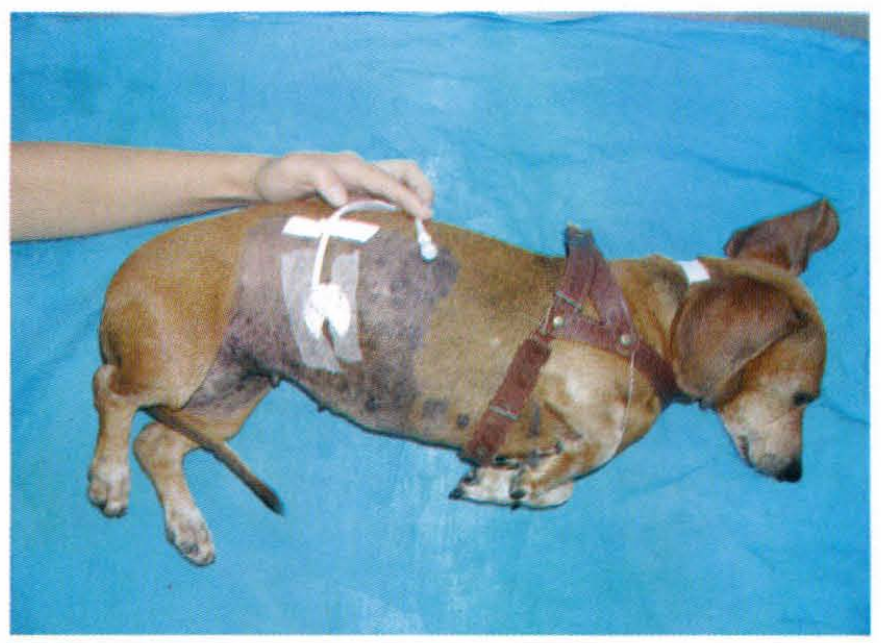

Figura 3 - Sonda gástrica aplicada em cão com estenose de esôfago 


\section{Conclusões}

A presente pesquisa demonstrou que a quantidade de energia metabolizável ingerida pelo animal está diretamente relacionada com sua alta, óbito e tempo de internação, demonstrando a importância do estabelecimento de um programa nutricional para o paciente.

\section{Agradecimentos}

ANFALPET - Associação Nacional dos Fabricantes de Alimentos para Animais de Estimação Mogiana Alimentos S.A. (Guabi)

\section{Abstract}

Objective: To investigate the correlation between caloric ingestion, hospital discharge and death rate of dogs and cats. Material and Method: Animals hospitalized at the Hospital Veterinário da Faculdade de Ciências Agrárias e Veterinárias da Universidade Estadual Paulista (HV-FCAV/UNESP), (Veterinarian Hospital of the College of Agrarian and Veterinarian Sciences of Paulista State University) Campus of Jaboticabal, SP, for any disease or in pre or post surgery period, had their maintenance energy requirement (MER): $132 x$ (body weigh) $)^{0.75}$ kc a day for dogs; $70 x$ (body weigh) kcal a day for cats. Animals were fed commercial superpremium food and received enteral and parenteral nutrition support. A total of 279 animals were studied, 223 dogs and 56 cats. Results: Were discharged from hospital 76,7\% of patients, the average percentage ingestion of (MER) of those animals was $71,12 \%$, while those who died ingested only $33.97 \%$ of $(M E R)(p<0,001)$. Animals of the Discharge group remained 7.18 days in hospital, while those in the group that died 5.45 days $(p=0,16)$. Among the animals that received from $0 \%$ to $33 \%$ of $M E R, 38 \%$ were discharged and $62 \%$ died, out of those who received $34 \%$ to $66 \%$ of MER, $84 \%$ were discharged and $16 \%$ died and for those who received more than $67 \%$ of MER, $89 \%$ of the animals were discharged and $11 \%$ died, indicating lower mortality rate in the group with higher caloric ingestion $(p<0,001)$. In the groups that had higher caloric ingestion, animals remained in hospital longer $(p<0,002)$, what can also be verified by the positive association between caloric ingestion and hospitalization lenght $(R=0,21 ; p<0,05)$. For the animals discharged from the hospital (76.7\% of the total), the average ingestion was $71.12 \%$ of the MER, and $11 \%$ died, which indicates a lower mortality rate for higher caloric balance $(p<0.001)$. For animals with higher caloric balance, the hospitalization lenght was longer $(p<0.001)$, which can be also verified from the positive association between caloric ingestion and hospitalization lenght $(R=0,21 ; p<0,05)$. Conclusions: Among the animals that we are able to fed larger quantities of calories, there were larger rates of hospital discharge and longer hospitalization stay.

Keywords: Hospitalization. Lenght of stay. Patient discharge. Clinical nutrition. Nutritional assessment. Energy requirement. Caloric intake. Dogs. Cats. 


\section{Resumen}

Objetivo: Investigar la correlación entre ingestión calórica y tiempo de internación con la alta de hospitalización o el óbito de perros y gatos. Material y Método: Animales internados en el Hospital Veterinario de la Facultad de Ciencias Agrarias y Veterinarias de la Universidad Estadual Paulista (HV-FCAV/UNESP), Campus de Jaboticabal, SP, Brasil, que por cualquier motivo o enfermedad sea en el pre o posquirúrgico, tuvieron estimada su necesidad energética de manutención (NEM): $132 x$ (peso corporal) ${ }^{0,75}$ kcal por día en perros; $70 x$ (peso corporal) kcal por día para gatos. Recibieron alimento comercial super-premium, soporte enteral y parenteral. Fueron incluidos 279 animales (233 perros y 56 gatos). Resultados: Tuvieron alta en el hospital el $76,7 \%$ de los pacientes, la ingestión porcentual media de la NEM de esos animales fue de 71,12\%, ya los que murieron ingirieron apenas 33,97\% de la NEM $(p<0,001)$. Los animales del grupo Alta permanecieron 7,18 días hospitalizados y los del grupo Óbito solamente 5,45 días $(p=0,16)$. Dentro del grupo de animales que recibieron de 0\% a 33\% de la NEM, el 38\% tuvo alta y el 62\% murió, para los que recibieron entre un 34\% a un $66 \%$ de la NEM, $84 \%$ tuvieron alta y el 16\% murió y para los que recibieron más que $67 \%$ de la NEM, $89 \%$ de los animales tuvieron alta y un $11 \%$ murió, demostrando menor mortalidad en los intervalos de mayor equilibrio calórico $(p<0,001)$, En los intervalos de mayor equilibrio calórico el tiempo de hospitalización fue mayor ( $p<0,002)$, esto se ratifica por la asociación positiva entre la ingestión de calorías y días de internación $(R=0,21 ; p<0,05)$. Conclusiones: Se verificó que para los animales a los que se consiguió administrar mayor cantidad de calorías hubo un aumento del tiempo de hospitalización y de las altas de hospitalización.

Palabras-clave: Hospitalización. Tiempo de internación. Alta del paciente. Terapia nutricional. Evaluación nutricional. Necesidad energética. Ingestión de energía. Perros. Gatos.

\section{Referências}

1. REMILlARD, R. L. et al. Assisted feeding in hospitalization patients: enteral and parenteral nutrition. In: HAND, M.S. et al. Small animal clinical nutrition. 4. ed. Topeka: Mark Morris Institute, 2000. p. 351-400.

2. REMILLARD, R. L. et al. An investigation of the relationship between caloric intake and outcome in hospitalization dogs. Veterinary Therapeutics, v. 2, p. 301-310, 2001.

3. BOULCOTT, S. R. The feeding behaviour of adults dogs under conditions of hospitalization. British Veterinary Journal, v. 123, p. 498-507, 1967.
4. BUTTERWICK,R. F.; TORRANCE, A. Nutrición y malnutrición en los pequeños animales hospitalizados. Walthan Focus, v. 5, n. 2, p.15-21, 1995.

5. SEIM III, H. B.; BARTGES, J. W. Enteral and parenteral nutrition. In: TAMS, T.T. Handbook of small animal gastroenterology. Saint Louis: Saunders, 2003. p. 416-462.

6. BATTAGLiA, A. M. Nutrition for the critically ill hospitalized patient. In: Small animal emergency and critical care: a manual for the veterinary technician. New York: Saunders, 2001. p. 72-93. 
7. DEVEY, J. J. et al. Postsurgical nutritional support. Journal of the American Veterinary Medical Association, v. 206, p.1673-1675, 1995.

8. CASE, L. P., CAREY, E. P., HIRAKAWA, D. A. Canine and feline nutrition: a resource for companion animal professionals. Saint Louis: Mosby, 1995. $455 \mathrm{p}$.

9. NATIONAL RESEARCH COUNCIL. Nutrient requirements of cats. Washington, DC: National Academy Press, 1986.

10. NATIONAL RESEARCH COUNCIL. Nutrient requirements of dogs. Washington, DC: National Academy Press, 1985.

11. KELLY, N. C. Food types and evaluation. In: KELLY, N.C.; WILLS, J. BSAVA manual of companion animal nutrition and feeding. Ames: Blackwell, 1996. p. 22-42.

12. KLEIBER, M. Body size and metabolism. Hilgardia, v. 6, p. 315, 1932.

13. SCHLOTZHAUER, S.; LiTTEll, R. C. SAS System for elementary statistical analysis. 2. ed. Cary: SAS Institute, 1997. p. 456.

14. BURKHOLDER, W. J. Metabolic rates and nutrients requirements of sick dogs and cats. Journal of the American Veterinary Medical Association, v. 206, p. 614-618, 1995.

15. CHANDRA, R. K. Immunodeficiency in undernutrition and overnutrition. Nutritional Reviews, v. 39, p. 225-231, 1981.

16. CRANE, S. W. Nutritional aspects of wound healing. Seminary of Veterinary Medicine Surgery, v. 4, p. 263-267, 1989.

17. CHANDRA, R. K. Nutrition and immunoregulation: significance for host resistence to tumors and infectious diseases in humans and rodents. Journal of Nutrition, v. 122, p. 754-757, 1992. Supplement 3.
18. CHANDRA, R. K. Protein energy malnutrition and immunological responses. Journal of Nutrition, v. 122, p. 597-600, 1992. Supplement 3.

19. FETTMAN, M. J.; PHILliPS, R. W. Dietary effects on drugs metabolism. In: HAND, M.S. et al. Small animal clinical nutrition. 4. ed. Topeka: Mark Morris Institute, 2000. p. 924-939.

20. SIMPSON, J. W. et al. Anorexia, enteral and parenteral feeding. In: SIMPSON, J. W. et al. Clinical nutrition of the dog and cat. Oxford: Blackwell, 1994. p. 96-114.

21. LEWIS, L. D. et al. Anorexia, inanition, and critical care nutrition. In: HAND, M.S. et al. Small animal clinical nutrition. 3. ed. Topeka: Mark Morris Institute, 1990. p. 5.1-5.43.

22. HILL, R. C. Critical care nutrition. In: WILLS, J.M. ; SIMPSON, K.W. The Walthan book of clinical nutrition of dog and cat. Oxford: Pergamon, 1994. p. 39-61.

23. TENNANT, B. Feeding the sick animal. In: KELLY, N.C.; WILLS, J. BSAVA manual of companion animal nutrition and feeding. Ames: Blackwell, 1996. p. 181-187.

24. JOHANSEN, N. et al. Effect of nutritional support on clinical outcome in patients at nutritional risk. Clinical Nutrition, v. 22, p. 85-86, 2003. Supplement 1.

25. CARNEVALE, J. M. et al. Nutritional assessment: guidelines to selecting patients for nutritional support. Compendium on Continuing Education for the Practicing Veterinarian, v. 13, p. 255-261, 1991.

26. SAKER, K. E. Diet and the immune system: selected overview of nutritional immunomodulation. In: PETFOOD FORUM, 2004, Chicago. Proceedings... Chicago: Petfood Industry, 2004. p. 44-59.

27. CROWE, D. T. et al. The use of polymeric liquid enteral diets for nutritional support in seriously ill or injured small animals: clinical results in 200 patients. Journal of the American Animal Hospital Association, v. 33, p. 500-508, 1997. 
28. DONOGHUES, S.; KRONFELD, D. S. Feeding hospitalized dogs and cats. In: WILLS, J.M.; SIMPSON, K.W. The Walthan book of clinical nutrition of dog and cat. 1994. p. 25-37.

29. SIMPSON, K. W.; ELWOOD, C. M. Techniques for enteral nutrition suport. In: WILLS, J.M.; SIMPSON, K.W. The Walthan book of clinical nutrition of dog and cat. Oxford: Pergamon, 1994. p. 63-74.

30. ABOOD, S. K.; BUFFINGTON, C. A. Improved nasogastric intubation technique for administration of nutritional support in dogs. Journal of the American Veterinary Medical Association, v. 199, p. 577 $579,1991$.

31. ABOOD, S. K.; BUFFINGTON, C. A. Enteral feeding of dogs and cats: 51 cases (1989-1991). Journal of the American Veterinary Medical Association, v. 201, p. 619-622, 1992.

32. LEVINE, P. B. et al. Esophagostomy tubes as a method of nutritional management in cats: a retropective study. Journal of the American Animal Hospital Association, v. 33, p. 405-410, 1997. 